\title{
2.3. THE DIRECTION OF THE MINOR AXIS OF GEODETIC REFERENCE SPHEROIDS
}

\author{
Brigadier G. Bomford and DR. A. R. Robbins \\ (Oxford University, Oxford, England)
}

\begin{abstract}
It is necessary to standardise the definition of the direction of the minor axes of spheroids used for geodetic computations. In view of polar motion, the definition "parallel to the axis of rotation" is ambiguous. Few countries have explicitly stated the direction adopted, and it has generally been implicitly defined by the corrections for polar motion applied to astronomical azimuths, latitudes, and longitudes. But these corrections have often been neglected or applied inconsistently, and errors of perhaps 0 . 5 of azimuth result. The choice for a recommended Geodetic Mean Pole, to which spheroidal minor axes can in future be defined to be parallel, seems to lie either with the Cecchini "new system, 1900-05" or with the Mean Pole of Epoch 1962.0.
\end{abstract}

\section{RÉSUMÉ}

Il est nécessaire qu'on unifie la définition de la direction des petits axes des sphéroïdes utilisés pour les calculs géodésiques. En considération du mouvement du pôle, la définition "parallèle à l'axe de la rotation" est ambigue. Peu de services géodésiques ont affirmé explicitement la direction adoptée, et ordinairement on l'a définie implicitement par les corrections pour le mouvement du pôle appliquées aux azimuts, latitudes et longitudes astronomiques. Mais on a négligé souvent ces corrections, ou on les a appliquées illogiquement, et il résulte des erreurs peut-être de 0 ". 5 d'azimut. Le choix d'un Pôle Moyen Géodésique recommandé, par rapport auquel à l'avenir on peut prendre parallèles les petits axes des sphéroïdes, semble de reposer soit avec le Cecchini "new system, 1900-05", soit avec le Pôle Moyen de l'Epoque de 1962.0.

\section{Notation}

Geodetic A direction, fixed in relation to the Earth, to which the minor axes of Mean Pole geodetic reference spheroids are to be parallel; denoted by a defined point on polar motion diagrams.

$x_{1}, y_{1} \quad$ Coordinates of the instantaneous pole with reference to the Geodetic Mean Pole as in Figure 1 (seconds of arc).

$x_{2}, y_{2} \quad$ Coordinates of the BIH mean pole of epoch with reference to the Geodetic Mean Pole (seconds of arc).

$\phi_{\mathrm{G}}, \lambda_{\mathrm{G}}, A_{\mathrm{G}}$ Geodetic latitude, longitude and azimuth at a field station.

$\phi_{\mathrm{A}}, \lambda_{\mathrm{A}}, A_{\mathrm{A}} \quad$ Astronomical latitude, longitude and azimuth at a field station.

$\phi, \lambda \quad$ Approximate latitude and longitude at a field station.

$\phi_{\mathrm{b}} \quad$ Astronomical latitude of a BIH observatory.

$\lambda_{\mathrm{b}} \quad$ Adopted astronomical longitude of a BIH observatory.

Markowitz and Guinot (eds.), Continental Drift, 37-43. () I.A.U. 
$w_{\mathrm{b}} \quad$ Weight of a BIH observatory. All latitudes positive north and longitudes positive east (astronomers use positive west).

$\mathrm{LST}_{\mathrm{a}} \quad$ Local sidereal time at a field station.

$\mathrm{LST}_{\mathrm{b}} \quad$ Local sidereal time at a BIH observatory.

$R$ Greenwich apparent sidereal time at $0^{\mathrm{h}}$ U.T.

$90^{\circ} \mathrm{W}$
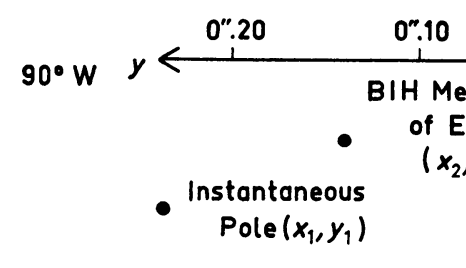

- Cecchini's Origin

1900-05

Adopted Geodetic

Mean Pole $(0,0)$

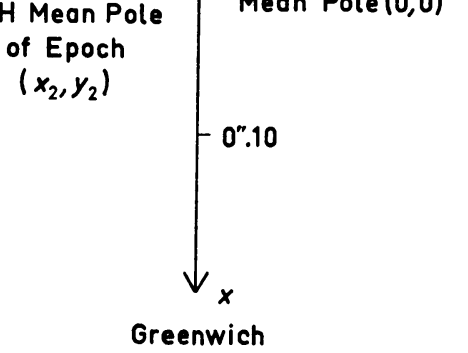

FIG. 1. Note that the relative positions of the various poles are diagrammatic only.

\section{Geodetic Reference Systems}

Geodesists compute positions in terms of latitude and longitude on a reference spheroid. For every detached survey they adopt arbitrary values for the major axis and flattening. They define the centre of the spheroid with reference to the direction of the vertical at some station known as their origin. Finally, they have to define the direction of the minor axis of the spheroid, and the direction of the zero meridian plane. In this paper we are only concerned with the last two items.

Loosely, the minor axis is always defined to be parallel to the Earth's axis of rotation. But if accepted geodetic latitudes and longitudes are to be constant, as they generally must be, the minor axis must be fixed in the rigid body of the Earth. Consequently, in view of the existence of Polar Motion, the minor axis of the spheroid cannot be defined as parallel to the instantaneous axis of rotation. The definition must either place it parallel to the line of points in the Earth which was the axis of rotation on some specified date, or else must refer to some mean position related to past directions of that axis.

The observed motion of the axis of rotation, or of the celestial pole, relative to the Earth, is customarily shown in a coordinate system such as that illustrated in Figure 1, which is of course fixed in relation to the Earth and shares its precession and nutation. Then the direction of the minor axis of the geodetic spheroid may be defined by designating any point on such a diagram as the adopted Geodetic Mean Pole. 
When computations are carried out in Cartesian coordinates $X, Y, Z$, the direction of the $Z$-axis must be similarly defined, and the $X$-axis should lie in the Geodetic Zero Meridian, as defined in Section 5 below.

\section{Formulae for Azimuth, Latitude and LST}

At first sight a change in the direction of the minor axis, say $\theta^{\prime \prime}$, would constitute a rotation which would change geodetic latitudes by comparable amounts, but in practice the geodetic latitude and longitude at the origin of any survey are held fixed (while the centre of the spheroid may be moved), so that the changes are much smaller, especially in a survey covering only a small area. The effect of changing the axis is correctly given if astronomical observations of latitude, longitude and azimuth are corrected as in equations (1)-(7) below.

An astronomical azimuth, observed with reference to the instantaneous axis of rotation, requires correction to the Geodetic Mean Pole as below.

Azimuth (mean)-Azimuth (Inst)=

$$
-\left(x_{1} \sin \lambda+y_{1} \cos \lambda\right)^{\prime \prime} \sec \phi .
$$

Similarly the correction to an observed astronomical latitude is

$$
+\left(y_{1} \sin \lambda-x_{1} \cos \lambda\right)^{\prime \prime} .
$$

And the correction to an observed LST is

$$
-\frac{1}{15}\left(x_{1} \sin \lambda+y_{1} \cos \lambda\right) \tan \phi \text { seconds. }
$$

\section{Longitude and Laplace Azimuths}

The correction to an observed astronomical longitude is complicated by the fact that U.T. 1 is reduced by the BIH to a variable Mean Pole of Epoch which in general cannot exactly coincide with the fixed Geodetic Mean Pole.

If all observations were reduced to the same pole we would have

$$
\begin{aligned}
\lambda_{\mathrm{A}} & =\mathrm{LST}_{\mathrm{a}}-R-\text { U.T. } 1 \\
& =\mathrm{LST}_{\mathrm{a}}-R-\frac{1}{\Sigma w_{\mathrm{b}}} \sum w_{\mathrm{b}}\left(\mathrm{LST}_{\mathrm{b}}-R-\lambda_{\mathrm{b}}\right),
\end{aligned}
$$

where the summation includes all observatories contributing to U.T. 1,

$$
=\mathrm{LST}_{\mathrm{a}}-\frac{1}{\Sigma w_{\mathrm{b}}} \sum w_{\mathrm{b}}\left(\mathrm{LST}_{\mathrm{b}}-\lambda_{\mathrm{b}}\right) \text {. }
$$


In equation (4), even though the field $\mathrm{LST}_{\mathrm{a}}$ may have been corrected from instantaneous to some fixed pole by (3), the $\mathrm{LST}_{\mathrm{b}}$ 's will have been corrected by the BIH to the moving mean pole of epoch, and will each require the further correction

$$
-\frac{1}{15}\left(x_{2} \sin \lambda_{\mathrm{b}}+y_{2} \cos \lambda_{\mathrm{b}}\right) \tan \phi_{\mathrm{b}} .
$$

So the total correction to a field longitude computed with U.T. 1 is

$$
\begin{aligned}
& -\frac{1}{15}\left(x_{1} \sin \lambda+y_{1} \cos \lambda\right) \tan \phi \\
& +\frac{1}{15 \Sigma w_{b}} \sum w_{b}\left(x_{2} \sin \lambda_{b}+y_{2} \cos \lambda_{b}\right) \tan \phi_{b} .
\end{aligned}
$$

The geodetic Laplace azimuth at a field station is given by

$$
A_{\mathrm{G}}=A_{\mathrm{A}}-\left(\lambda_{\mathrm{A}}-\lambda_{\mathrm{G}}\right) \sin \phi,
$$

in which $A_{\mathrm{A}}$ requires correction as in (1), and $\lambda_{\mathrm{A}}$ as in (6), and the total correction to $A_{\mathrm{G}}$ is

$$
\begin{aligned}
-\left(x_{1} \sin \lambda+y_{1} \cos \lambda\right) \cos \phi & \\
& -\frac{\sin \phi}{\Sigma w_{\mathrm{b}}} \sum^{w_{\mathrm{b}}}\left(x_{2} \sin \lambda_{\mathrm{b}}+y_{2} \cos \lambda_{\mathrm{b}}\right) \tan \phi_{\mathrm{b}} .
\end{aligned}
$$

For the 46 observatories used by the BIH in 1966 the value of $1 /\left(\sum w_{b}\right) \sum\left(x_{2} \sin \lambda_{b}+\right.$ $\left.y_{2} \cos \lambda_{\mathrm{b}}\right) \tan \phi_{\mathrm{b}}$ is

$$
0 \cdot 29 x_{2}^{\prime \prime}+0.42 y_{2}^{\prime \prime} .
$$

The factor 0.42 is larger than might be expected, by reason of the uneven distribution of the observatories.

\section{Zero Meridian Plane}

The BIH zero meridian is a plane, containing the BIH Mean Pole of Epoch, through which the First Point of Aries passes at U.T. $1=24^{\mathrm{h}}-R$. If all BIH observed LST's are corrected to the adopted Geodetic Mean Pole as in (5), the resulting Geodetic Zero Meridian will lie East of the BIH zero by

$$
-\frac{1}{\sum w_{\mathrm{b}}} \sum w_{\mathrm{b}}\left(x_{2} \sin \lambda_{\mathrm{b}}+y_{2} \cos \lambda_{\mathrm{b}}\right) \tan \phi_{\mathrm{b}} .
$$

This zero meridian will be the zero of both geodetic and astronomical longitudes, $\lambda_{\mathrm{G}}$ and $\lambda_{\mathrm{A}}$, as used by geodesists. 


\section{Magnitude of Errors}

In equations (1)-(8) $x_{1}$ and $y_{1}$ vary about an annual mean position by not more than $0^{\prime \prime} .3$ in a year of large polar motion. If the adopted Geodetic Mean Pole is the Cecchini 1900-05 origin, $y_{1}$ may in the 1960's reach 0".5. The result of ignoring Equations (1)-(7) may then possibly be as much as 0 ".5, but generally not more than $0^{\prime \prime} .2$ or $0 " .3$ ( $\times \tan \phi$ for longitudes). Errors arising from the 12- or 14-month motion will be more or less random, but the difference between adopting one mean pole (e.g. 1962) and another (e.g. 1903) may be a systematic 0 ".2. The most serious consequence of neglecting the corrections is that Laplace azimuths may be wrong by these amounts. The corrections to astronomical latitudes and longitudes also cause changes in the deviations of the vertical, which affect geoidal sections and thence the reduction of measured distances to spheroid level, but in a survey extending less than (say) $4000 \mathrm{~km}$ from its origin this effect will be a few times less than that of the errors of azimuth.

\section{Current Practice}

In the past, few if any surveys have explicitly stated the defined direction of their minor axis, and there has been little or no consistency in the definitions implicitly made by reductions to Mean Pole. Some countries may have consistently applied equation (1) to observed astronomical azimuths, but it is more doubtful whether any have correctly reduced their Laplace azimuths. Possibly the nearest approach to consistency is when some country has used equations (1), (2) and (3) to reduce its observations to the BIH Mean Pole of Epoch. Then if its observations have not extended over many years, it can claim to have been nearly consistent, using the mean BIH mean pole over the period.

Writing in January 1967 we have not got full information about which countries may have been consistent, or nearly so.

\section{Change of Spheroid}

When detached surveys are computed with different spheroids and origins with (inevitably) different centres, a trustworthy common point makes it possible to reduce the latitudes and longitudes given by one into terms of the other. If the two surveys have parallel axes, the conversion is simple. If they have not, it is less simple.

\section{Star Catalogues}

Random probable errors, when brought up to the present epoch, are estimated to be of the order 0 ". 2 for FK4, 2" for the Boss G. C. In both cases some stars, in particular those of high Southern declination, are subject to larger errors than are generally 
applicable, and in addition there will be considerable systematic error. Current observations should be computed with FK4, the catalogue on which the observations used by the BIH are based.

\section{Future Needs}

Errors such as those mentioned in Section 6 are less than the normal errors of observation: in Laplace azimuths about half the usual probable error or less. It is unlikely that any existing survey is materially the worse for their neglect.

It is to be expected, however, that satellite triangulation and other new techniques will increase accuracy, and now is the time to agree on a Mean Pole which should be adopted for future international geodetic work. We may hope that we are not acting too late, but little more delay can be afforded.

If any considerable geodetic computations had been consistently carried out using any particular mean pole, we would now be inclined to secure agreement to its adoption by all. Australia may be such a case. It is possible that there are others, but it is thought that there is unlikely to be any conflict between existing interests, and that we have a fairly free choice. What cannot be adopted is a continuously moving "Mean Pole of Epoch".

The choice seems to lie between two possible Mean Poles.

(a) The Cecchini "new system 1900-05". This is about 0".2 distant from the present position of the mean. If there is any doubt about that figure, it would not be expedient to define the Geodetic Mean Pole as coinciding with the actual mean of 1900-05, but it could quite properly be defined as the origin of the IPMS polar coordinates. Then the current positions of the pole can be regarded as correct, while the geodetic mean pole is defined to be the zero point which gives them their accepted coordinates. The position of the pole 50 years ago might remain doubtful.

A great many individual observations have no doubt been reduced to this Cecchini origin, but it is doubtful whether any large survey, currently accepted and not soon due for recomputation, has been consistently computed in terms of it.

(b) The mean pole of epoch 1962.0. If a more recent position is to be accepted, this is a convenient date, since it coincides with the introduction of the FK4 catalogue and the new adopted longitudes in the work of the BIH. It avoids an unnecessary systematic correction of about 0.2 in modern work, and it has just been accepted for Australia.

Subject to the comments of all those interested, it is thought likely that the mean pole of epoch 1962.0 will be found to be the most convenient definition of the Geodetic Mean Pole.

\section{Action Now Required}

Delegates at the Stresa symposium are asked to recommend a fixed Mean Pole for future geodetic reference systems. 


\section{Nomenclature}

We have used the expressions "Geodetic Mean Pole" and "Geodetic Zero Meridian", as is necessary so long as other interests use a moving Pole. If a convenient fixed Pole and zero meridian could be adopted by all, there would be no need to define it as "Geodetic". It would suffice for geodesists to recommend its adoption for their work. 